\title{
Observation of positive and negative trions in organic-inorganic hybrid perovskite nanocrystals
}

\author{
Naoki Yarita, Tomoko Aharen, Hirokazu Tahara, Masaki Saruyama, Tokuhisa Kawawaki, \\ Ryota Sato, Toshiharu Teranishi, and Yoshihiko Kanemitsu* \\ Institute for Chemical Research, Kyoto University, Uji, Kyoto 611-0011, Japan
}

(Received 3 August 2018; revised manuscript received 1 October 2018; published 14 November 2018)

\begin{abstract}
Lead halide perovskite nanocrystals are promising light emitting materials with high photoluminescence quantum yields and widely tunable emission wavelengths. Their optical responses are significantly influenced by efficient nonradiative Auger recombination of trions (charged excitons) and biexcitons. Here, we report the observation of positive and negative trions in formamidinium lead bromide nanocrystals and discuss their role in the photoluminescence dynamics. It is found that the addition of copper thiocyanate causes an additional intermediate state by photochemical doping. We clarify that as-prepared nanocrystals show a two-state blinking between neutral excitons and positive trions, while the postsynthesis treated nanocrystals exhibit blinking between all three states, including negative trions. We confirmed that the biexciton Auger rate evaluated from femtosecond transient absorption measurements can be expressed as the sum of the Auger rates of positive and negative trions obtained by single-dot spectroscopy.
\end{abstract}

DOI: 10.1103/PhysRevMaterials.2.116003

\section{INTRODUCTION}

Very recently, there have been extensive studies on optical and transport properties of the lead halide perovskites $A \mathrm{~Pb} X_{3}$ $\left(A=\mathrm{CH}_{3} \mathrm{NH}_{3}, \mathrm{HC}\left(\mathrm{NH}_{2}\right)_{2}, \mathrm{Cs} ; X=\mathrm{Cl}, \mathrm{Br}, \mathrm{I}\right)$, because they are a class of promising materials for optoelectronic devices such as thin-film solar cells [1,2], light sources [3-5], and optical switches [6]. Substantial progress has been made in clarifying the origin of their excellent electronic properties, such as long carrier lifetimes [7,8], long carrier diffusion lengths [9-12], and photon recycling [13]. One of the greatest advantages of perovskite-based optoelectronic devices is that the active layer can be fabricated by simple solution-based methods. Since the report of a lead halide perovskite solar cell in 2009 [14], the record conversion efficiency of the perovskite solar cells shows continuous growth every year, and is now at $23.3 \%$ [15]. In addition, the external efficiency of perovskite-based light-emitting diodes (LEDs) has recently increased above $10 \%$ [16]. These high efficiencies are originated from the perovskite's almost ideal electronic structure in which deep trap levels are rarely formed, because of their unique characteristics of chemical bonds and band structures [17-19].

By reducing its crystal size to several nanometers, the photoluminescence (PL) quantum yield (PLQY) can be further enhanced via quantum confinement [20]. To take advantage of quantum confinement effects in halide perovskites,

\footnotetext{
*Author to whom correspondence should be addressed: kanemitu@scl.kyoto-u.ac.jp

Published by the American Physical Society under the terms of the Creative Commons Attribution 4.0 International license. Further distribution of this work must maintain attribution to the author(s) and the published article's title, journal citation, and DOI.
}

all inorganic $\mathrm{CsPb} X_{3}$ nanocrystals (NCs) were successfully synthesized in 2015 [21]. By replacing the halide ions, the band-gap energy can be adjusted within the entire visible range to obtain desired luminescence colors $[21,22]$. Under weak excitation conditions, perovskite NCs show very high PLQYs of up to $90 \%$ despite the simple synthesis method without any special surface passivation [21]. In the case of the well-studied II-VI semiconductors, it is difficult to achieve such a high PLQY without employing core/shell structures to protect the NC surface [23,24]. Although halide perovskites have a very large density of point defects, their electronic and optical properties are tolerant to the point defects and surface states (so-called defect-tolerant semiconductors) $[19,25]$. In addition, perovskite NCs show PL with narrower spectral bandwidths in the entire visible spectral region and faster decays, compared with the conventional covalent semiconductor NCs [25-27]. Thus, perovskite NCs have several advantages for highly efficient lasers and LEDs because they are both cost effective and bright [28-30].

The device performance of NC-based LEDs and lasers, which require high carrier densities, is usually determined by fast nonradiative Auger recombination processes [31-33]. Previous studies revealed that nonradiative Auger recombination rates of trions (charged excitons) [26,34] and biexcitons $[26,34,35]$ are extremely high in perovskite NCs. Thus, deep understanding of the Auger recombination processes is the key to improving the efficiencies of optoelectronic devices that implement these NCs. In particular, it has been found that trions are generated in perovskite $\mathrm{NCs}$ even under relatively weak excitation conditions [26,36], causing PL blinking, i.e., a temporal fluctuation of the PL intensity at the single NC level [37,38]. This is considered to be related to the presence of the charged defects and surface states in ionic-lattice perovskite NCs. Controlling of PL blinking and $\mathrm{NC}$ charging has been one of the most important issues in semiconductor NC science and engineering, because the 
suppression of these fluctuations directly leads to improved PLQYs and high device performance of NC-based LEDs $[33,38]$. Furthermore, for laser applications, it has been shown that trions are useful for realization of low-threshold lasers [39-41]. Nevertheless, the research on trion formation and decay dynamics has not progressed sufficiently for halide perovskite NCs. The detailed investigation of trions and their recombination processes in the perovskite NCs is demanded for their optimization and implementation in advanced optoelectronics.

In this work, we report the observation of both positive and negative trions in single formamidinium lead bromide $\left(\mathrm{FAPbBr}_{3}\right) \mathrm{NCs}$ in films prepared with postsynthesis chemical treatments. For single NCs in films without any treatment, a two-state blinking between the high and low PL intensity states is clearly observed. By mixing copper thiocyanate $(\mathrm{CuSCN})$ into the films, the duration time at the state with the low PLQY is significantly reduced and an additional intermediate state appears. The analysis of the PL decays reveals that the low PL intensity state is caused by the positive trion and then the intermediate intensity state is assigned to the negative trion. We further demonstrate that the Auger rate of biexcitons is expressed as the sum of the Auger rates for positive and negative trions.

\section{EXPERIMENTAL}

\section{A. Samples}

The $\mathrm{FAPbBr}{ }_{3} \mathrm{NCs}$ were prepared according to the method described in the literature $[42,43]$. The procedure is briefly outlined below.

First, the mixture of oleylamine ( $12.5 \mathrm{~mL}$, Acros Organics) and ethanol $(100 \mathrm{~mL})$ was prepared, then a $47 \% \mathrm{HBr}$ aqueous solution $(8.56 \mathrm{~mL}, \mathrm{TCI})$ was slowly added to the ethanolic solution at $0^{\circ} \mathrm{C}$ under nitrogen flow. The solution was stirred at $0{ }^{\circ} \mathrm{C}$ for $12 \mathrm{~h}$. The subsequent solvent removal was done by evaporation. The obtained white precipitate (oleylammonium bromide) was washed three times with diethyl ether and dried under vacuum for $12 \mathrm{~h}$.

Next, lead acetate $(38.0 \mathrm{mg}$, Wako), formamidinium acetate (39.0 mg, TCI), dried 1-octadecene (4 mL, Sigma-Aldrich), and dried oleic acid ( $1 \mathrm{~mL}, \mathrm{TCI})$ were placed in a three-necked flask and dried under vacuum at $50^{\circ} \mathrm{C}$ for $30 \mathrm{~min}$. Then, the resultant mixture was heated to $130^{\circ} \mathrm{C}$ under nitrogen atmosphere. The homemade oleylammonium bromide $(105 \mathrm{mg})$ and toluene $(1 \mathrm{~mL})$ was injected, waited for $10 \mathrm{~s}$, and the solution was cooled in a water bath. Toluene $(5 \mathrm{~mL})$ and acetonitrile $(2.5 \mathrm{~mL})$ were added to the resulting solution at room temperature and the mixture was centrifuged at $8900 \mathrm{rpm}$ for $5 \mathrm{~min}$. The precipitate was recovered and redispersed in hexane. This hexane solution was again centrifuged at $8900 \mathrm{rpm}$ for $5 \mathrm{~min}$, and the precipitate was discarded to remove the large $\mathrm{FAPbBr}_{3}$ NCs. The optical absorption and PL spectra of our NC samples are shown in Fig. 1. The average edge length of the prepared NCs was $10.5 \pm 1.3 \mathrm{~nm}$ and the emission peak wavelength was $530 \mathrm{~nm}$ $(2.34 \mathrm{eV})$.

For post-synthesis treatment with copper thiocyanate $(\mathrm{CuSCN})$, the $\mathrm{FAPbBr}_{3} \mathrm{NCs}$ in hexane $(400 \mu \mathrm{L})$ was added

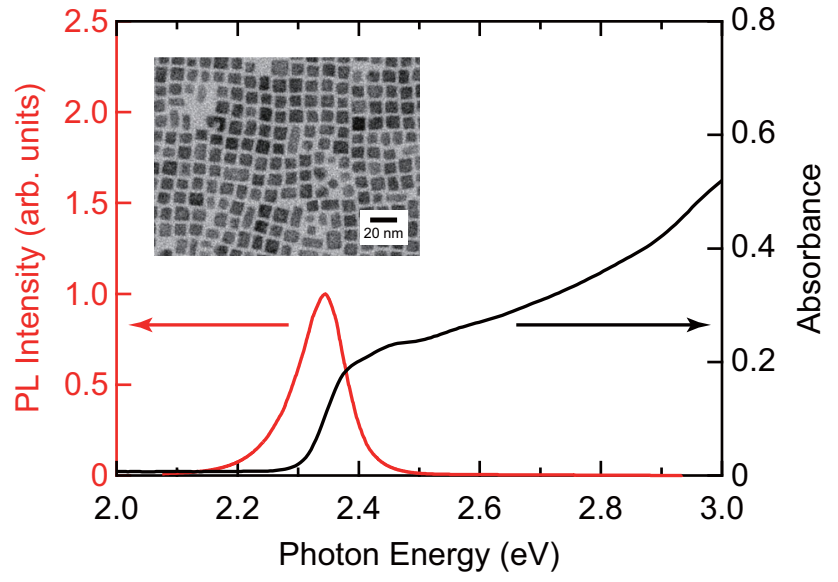

FIG. 1. Photoluminescence and absorption spectra of the $\mathrm{FAPbBr}_{3}$ nanocrystals dispersed in hexane. Transmission electron microscope image is shown in the inset.

to the copper thiocyanate $(50 \mathrm{mg})$ and stirred for $20 \mathrm{~min}$. Subsequently, the polymethylmethacrylate (PMMA) dissolved in toluene was added to the $\mathrm{NCs} / \mathrm{CuSCN}$ mixture to yield a suspension. The suspension was spin-coated onto a cover glass and used for single-dot spectroscopy.

\section{B. Single-dot optical spectroscopy}

We used a home-built confocal microscope system with a Hanbury Brown-Twiss interferometry setup to perform the second-order photon correlation studies on individual $\mathrm{FAPbBr}_{3} \mathrm{NCs}$. The NCs were excited at a wavelength of 441 $\mathrm{nm}$ with a bandwidth of $20 \mathrm{~nm}$ by means of a supercontinuum light source (WhiteLase, Fianiuum) combined with a tunable bandpass filter (SuperChrome, Fianiuum). The repetition rate of the excitation pulse was set to $5 \mathrm{MHz}$. To collect the emitted light from the sample, a $100 \times$ oil immersion objective with a numerical aperture of 1.3 for the visible range was used. We conducted all the measurements under ambient conditions.

The emitted light from the sample was divided with a beam splitter (transmission 50\%) and detected with a pair of identical silicon avalanche photodiodes (APDs) (ID Quantique). The detected signal on each APD was recorded on a respective time-correlated single-photon counting board (Becker \& Hickl) operating in the time-tag mode, which enables the simultaneous measurement of the PL decays and the secondorder photon correlation function $g^{(2)}$ with the time resolution of $\sim 240 \mathrm{ps}[44,45]$.

\section{RESULTS}

\section{A. Single-dot spectroscopy of $\mathrm{FAPbBr}_{3} \mathrm{NCs}$}

We performed time-tagged time-resolved PL (TTTR-PL) measurements on single $\mathrm{FAPbBr}_{3} \mathrm{NCs}$ with a bin time of $10 \mathrm{~ms}$. For single-dot spectroscopy, the colloidal dispersions were mixed with PMMA in toluene and spin-coated on a cover glass. The NC film was excited by a laser pulse (2.81-eV photon energy, $5-\mathrm{MHz}$ repetition rate, and $1.7-\mu \mathrm{J} / \mathrm{cm}^{2}$ excitation fluence). At $2.81 \mathrm{eV}$, the absorption cross section of these $\mathrm{FAPbBr}_{3} \mathrm{NCs}$ is 

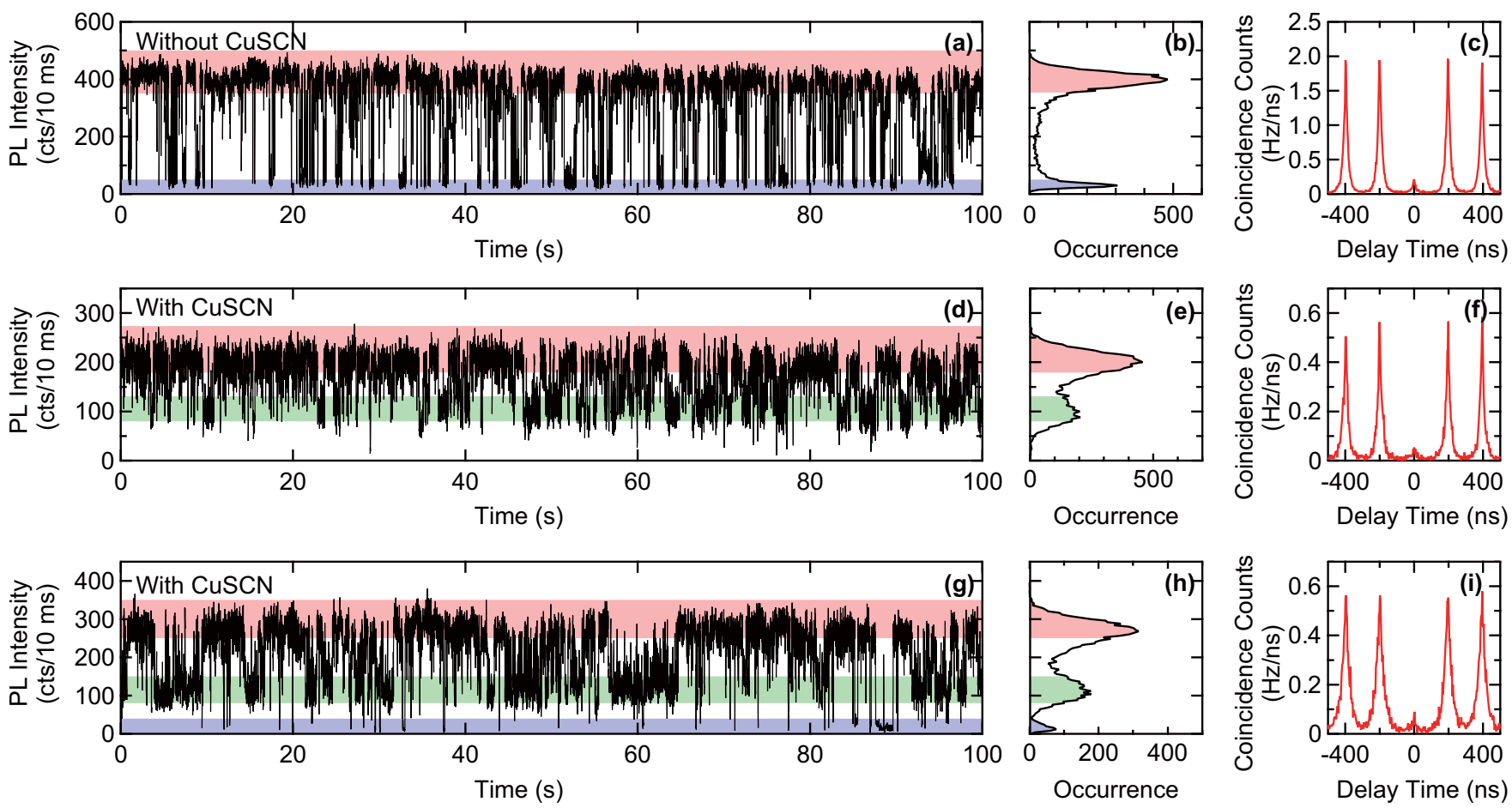

FIG. 2. (a), (d), (g) PL intensity time traces, (b), (e), (h) distribution of PL intensity and (c), (f), (i) second-order photon correlation function $g^{(2)}$ for (a)-(c) single $\mathrm{FAPbBr}_{3} \mathrm{NCs}$ without $\mathrm{CuSCN}$ and (d)-(i) with $\mathrm{CuSCN}$.

$3.4 \times 10^{-14} \mathrm{~cm}^{2}$ [43]. Thus, the average number of absorbed photons per $\mathrm{NC}$ and pulse, $\langle N\rangle$, is 0.1 .

Figure 2(a) shows a time trace of the PL intensity from a single $\mathrm{FAPbBr}_{3} \mathrm{NC}$ that exhibits discrete switching between the high PL intensity state (red area) and the low PL intensity state (blue area). These two states clearly appear in the distribution of the PL intensity shown in Fig. 2(b). The low PL intensity state in the perovskite's single NC emission originates from the emission of trions quenched by nonradiative Auger recombination [43]. Whether the charging of this trion is positive or negative can be clarified as discussed below. Figure 2(c) shows the second-order photon correlation function $g^{(2)}$ that was simultaneously measured for the same NC. The observed clear photon antibunching at zero delay time ensures that the signals shown in Figs. 2(a) and 2(b) are from a single NC. The small number of coincidence counts at zero delay time (center peak) is assigned to the twophoton cascade emission of biexcitons $[31,46]$. The area ratio between center peak and side peak (delay time $200 \mathrm{~ns}$ ) is 0.12 .

The addition of CuSCN to the $\mathrm{FAPbBr}_{3} \mathrm{NCs}$ dramatically changes the PL blinking behavior. $\mathrm{CuSCN}$ has been used in perovskite solar cells to extract photoexcited holes from the perovskite layer $[47,48]$. Thus, a negatively charged NC is formed upon addition of $\mathrm{CuSCN}$ due to the extraction of photogenerated holes from the NCs, i.e., CuSCN enables photochemical doping of perovskite NCs [49,50]. In Figs. 2(d), 2(g), 2(e), and 2(h), we show the time traces and PL intensity distributions, respectively, for two individual $\mathrm{FAPbBr}_{3} \mathrm{NCs}$ that were spin-coated using the perovskite NCs/CuSCN/PMMA suspension. Due to the presence of $\mathrm{CuSCN}$, an additional intermediate state appears, as indicated in green. We measured $45 \mathrm{NCs}$ in total (a variety of size and exciton lifetime shown in Appendix A) and almost all the measured NCs showed this intermediate state. Twentyeight out of the $45 \mathrm{NCs}$ showed a two-state blinking between the high and intermediate PL intensity states [Figs. 2(d) and 2(e)] and 15 NCs showed a three-state blinking between the high, intermediate, and low PL intensity states [Figs. 2(g) and 2(h)]. The trend observed here is that the evolution of the intermediate state is nearly concurrent with the disappearance of the PL low state. The remaining $2 \mathrm{NCs}$ exhibited a normal two-state blinking between the high and low PL intensity states like the NC shown in Figs. 2(a) and 2(b). The clear photon antibunching observed in the second-order photon correlation functions [Figs. 2(f) and 2(i)] confirms that these new types of blinking cannot be explained by a combined emission from two NCs. Since CuSCN is used to extract the photogenerated holes from NCs, the appearance of the intermediate state should be assigned to the negative trion in the single NC.

\section{B. PL decay dynamics of single NCs}

To investigate the origin of these three distinct states, we analyzed the PL decay dynamics of each state in all single NCs that exhibit the type of blinking shown in Fig. 2(g). Figure 3(a) shows the PL decay curve of the high PL intensity state, corresponding to the red shaded area in Fig. $2(\mathrm{~g})$. The PL decay curve can be fitted well using a single exponential function with a long lifetime of $21 \mathrm{~ns}$. The averaged exciton lifetimes are obtained to be 20.6 and 19.3 ns for $\mathrm{FAPbBr}_{3} \mathrm{NCs}$ with and without CuSCN, respectively (Fig. 5 in Appendix A). 

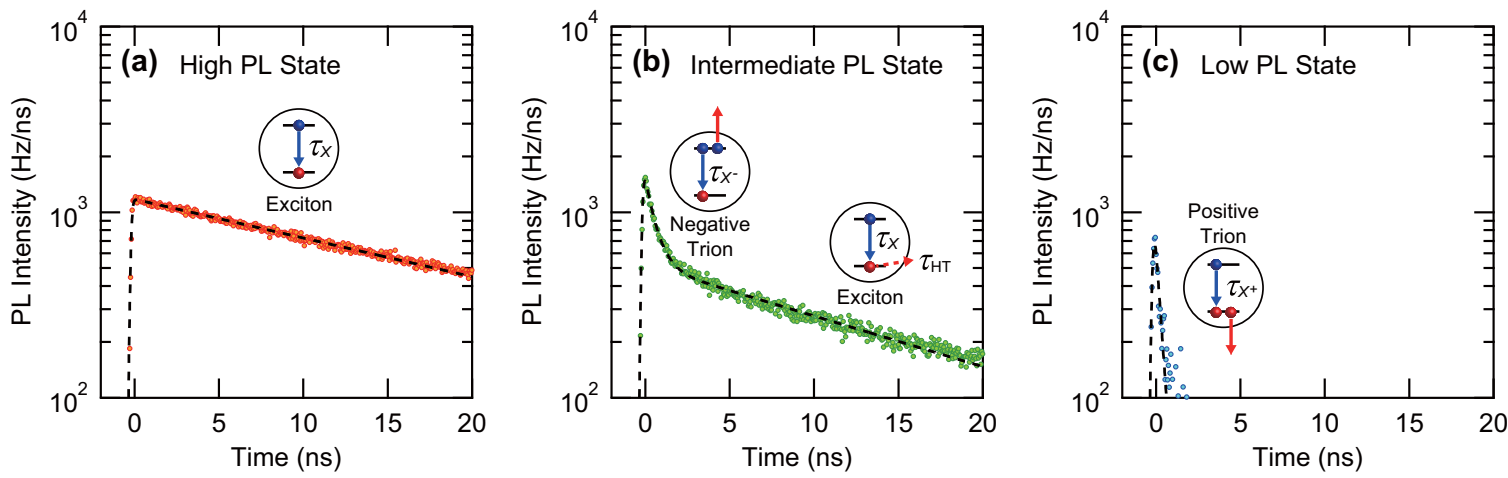

FIG. 3. (a)-(c) PL decay curve derived from the TTTR-PL data corresponding to (a) the red, (b) green, and (c) blue shaded areas in Figs. 2(g) and 2(h).

It is noteworthy that the exciton lifetime derived from the high PL intensity state shows no significant change upon addition of CuSCN. Therefore, it is concluded that the high PL intensity state originates from the exciton emission only, and hole extraction from the $\mathrm{NC}$ to the $\mathrm{CuSCN}$ is not influential on this state.

The PL decay curve of the intermediate PL intensity state exhibits a double exponential decay as shown in Fig. 3(b). The fast decay component (lifetime $=600 \mathrm{ps}$ ) can be assigned to Auger recombination of negative trions, which are generated upon hole extraction by $\mathrm{CuSCN}$. A similar intermediate state has been also observed in quasi-type-II band structure $\mathrm{CdSe} / \mathrm{CdS}$ core/shell NCs, and has been assigned to negative trions with a reduced Auger recombination rate [51-54]. However, our perovskite NCs have no shell structure, therefore, a different charging process, i.e., hole transfer to $\mathrm{CuSCN}$, should be considered. The hole transfer process can be analyzed with the slow decay component of the intermediate PL intensity state. The lifetime of this slow decay component is $16 \mathrm{~ns}$ for the same $\mathrm{NC}$, which is slightly shorter than the exciton lifetime determined for the high PL intensity state (21 ns). This slight difference between these lifetimes indicates that the PLQY of neutral excitons is reduced by the hole extraction to $\mathrm{CuSCN}$. Thus, we consider that two decay components of the intermediate PL intensity state correspond to two different processes: PL from negative trions and PL from neutral excitons with a reduced PLQY. The latter PL process is the dominant component determining the intermediate PL intensity, since the PLQY of negative trion is strongly reduced by Auger recombination (Fig. 6 in Appendix B).

The PL decay of the low PL intensity state shown in Fig. 3(c) contains a very fast component of $330 \mathrm{ps}$. This low PL intensity state was also observed for NCs without CuSCN in the film [Figs. 2(a) and 2(b)]. The histograms of the total time in the low PL intensity state are shown in Fig. 7 in Appendix C. Comparing the data with and without $\mathrm{CuSCN}$, it can be confirmed that the low PL intensity state is significantly suppressed by the addition of hole quencher CuSCN. This indicates that the low PL intensity state is derived from positive trions and the generation of the positive trion is suppressed due to the extraction of holes by CuSCN.

\section{DISCUSSION}

To verify our assignment of the CuSCN-triggered three-state blinking, we examined the correlation between the negative trion lifetime $\tau_{X^{-}}$and the positive trion lifetime $\tau_{X^{+}}$as plotted in Fig. 4(a). We found a lifetime ratio of $\tau_{X^{-}} / \tau_{X^{+}}=1.7$. From these two lifetimes, we can evaluate the biexciton lifetime $\tau_{X X}$ when we consider the number of recombination
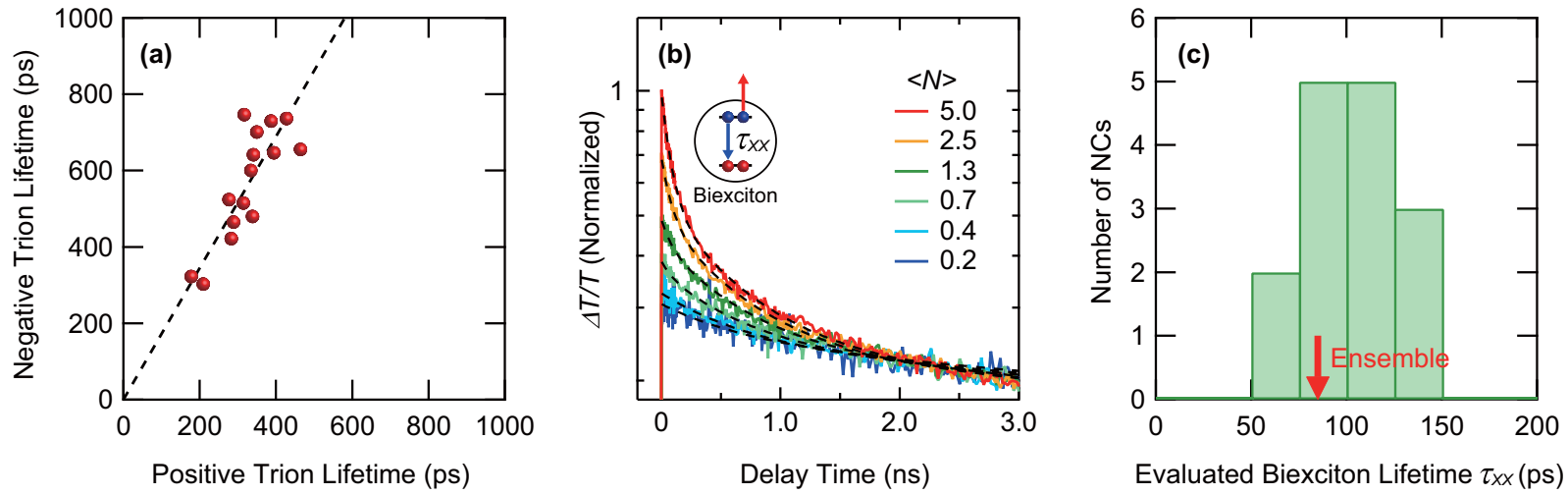

FIG. 4. (a) The relationship between the negative and positive trion lifetimes. The dashed line is the fitting result using a linear function. (b) Transient absorption dynamics of ensemble NCs dispersed in hexane. All decay curves are normalized at the late delay time of 3 ns. (c) Histogram of the biexciton Auger lifetime evaluated from the lifetimes of negative and positive trions. The red arrow indicates the biexciton Auger lifetime of the ensemble NCs obtained from panel (b). 
pathways. The biexciton Auger rate $\tau_{X X}{ }^{-1}$ can be treated as twice the sum of the two independent Auger rates for negative and positive trions: $\tau_{X X}{ }^{-1}=2\left(\tau_{X^{-}}{ }^{-1}+\tau_{X^{+}}{ }^{-1}\right)$ [54]. From this equation, the average value of the biexciton Auger lifetime is calculated to be 103 ps. Such a short lifetime is close to the resolution limit for our time-resolved single-dot spectroscopy setup since it has a time resolution of $\sim 240 \mathrm{ps}$. To obtain an accurate value for the biexciton Auger lifetime, therefore, we conducted femtosecond transient absorption spectroscopy on the $\mathrm{FAPbBr}_{3} \mathrm{NCs}$ dispersed in hexane. From the decay curves shown in Fig. 4(b), we obtained a biexciton Auger lifetime of $85 \mathrm{ps}$ in the ensemble sample, which agrees well with the average biexciton lifetime of 103 ps calculated from the individual lifetimes of negative and positive trions. In Fig. 4(c), the histogram data of the evaluated biexciton Auger lifetime for each single NC is compared with the biexciton Auger lifetime of the ensemble NCs (red arrow). This result supports our assignment of the intermediate state and the low PL intensity state to the negative and positive trions, respectively.

\section{CONCLUSION}

In conclusion, we determined the lifetimes of both positive and negative trions in single $\mathrm{FAPbBr}_{3} \mathrm{NCs}$. For assynthesized NCs, a two-state blinking between the high and low PL intensity states is observed. By mixing a hole quencher for perovskites, $\mathrm{CuSCN}$, the fraction of time in which the $\mathrm{NC}$ stays in the low PL intensity state is significantly reduced, indicating that the low PL intensity state is caused by generation of positive trions. Moreover, an additional intermediate state appears due to the addition of $\mathrm{CuSCN}$ to the film. The intermediate state is assigned to negative trions. Furthermore, we evaluated the Auger lifetimes of the biexcitons for individual NCs from the Auger lifetimes of positive and negative trions. The relationship between biexciton and trion lifetimes was clarified with transient absorption measurements on an ensemble sample. These observations show that the postsynthesis chemical treatment allows us to control the charge in perovskite NCs, i.e., positive and negative trions.

\section{ACKNOWLEDGMENT}

Part of this work was supported by JST-CREST (JPMJCR16N3).

\section{APPENDIX A: EXCITON LIFETIME}

Figure 5 displays the histograms of the exciton lifetime for the single $\mathrm{FAPbBr}_{3} \mathrm{NCs}$ with $\mathrm{CuSCN}$ (lower panel) and without (upper panel). We obtained averaged exciton lifetimes of 20.6 and $19.3 \mathrm{~ns}$, respectively. These lifetimes are close to the neutral exciton lifetime of $18 \mathrm{~ns}$, which we obtained for ensemble NCs dispersed in hexane without the CuSCN treatment.

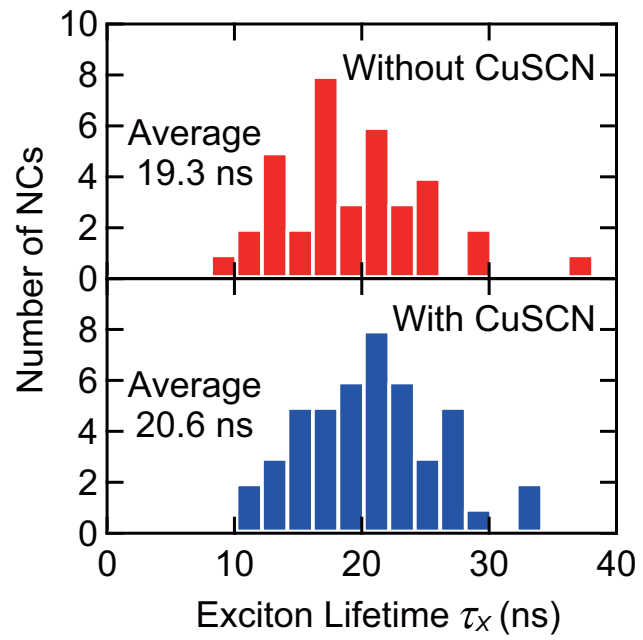

FIG. 5. Histograms of the exciton lifetime derived from the high PL intensity state for single NCs without (upper panel) and with CuSCN (lower panel).

\section{APPENDIX B: HOLE TRANSFER EFFICIENCY}

From the two exciton lifetimes in the high and intermediate PL intensity states, we can evaluate the hole transfer time $\tau_{\mathrm{HT}}$ and hole transfer efficiency $\eta_{\mathrm{HT}}$ with the following equations [55]:

$$
\begin{gathered}
\tau_{\mathrm{HT}}^{-1}=\tau_{X, \mathrm{Int}}{ }^{-1}-\tau_{X, \mathrm{High}}{ }^{-1}, \\
\eta_{\mathrm{HT}}=\frac{\tau_{\mathrm{HT}}^{-1}}{\tau_{X, \mathrm{High}}{ }^{-1}+\tau_{\mathrm{HT}}^{-1}}=1-\frac{\tau_{X, \mathrm{Int}}}{\tau_{X, \mathrm{High}}} .
\end{gathered}
$$

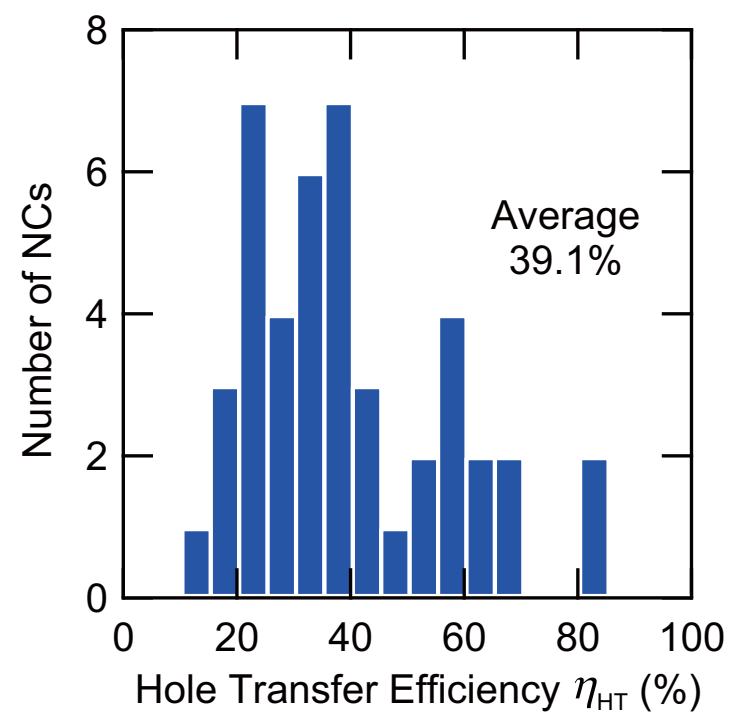

FIG. 6. Histogram of the hole transfer efficiency, $\eta_{\mathrm{HT}}$, derived from the high and intermediate PL intensity states for single NCs. 
Here, $\tau_{X, \text { Int }}$ is the slow lifetime derived from the intermediate state and $\tau_{X \text {,High }}$ is the exciton lifetime derived from the high PL intensity state. For the NC analyzed in Fig. 3(b), we obtain $\tau_{\mathrm{HT}}=67.1 \mathrm{~ns}$ and $\eta_{\mathrm{HT}}=23.7 \%$. Figure 6 shows the histogram of the hole transfer efficiency with an average hole transfer efficiency of $39.1 \%$. The variation of the hole transfer efficiency suggests that the hole transfer efficiency reflects the distance and the band offset between CuSCN and the NCs. This moderate transfer efficiency allows us to observe the coexistence of the high and intermediate PL intensity states in the PL blinking shown in Fig. 2(g).

\section{APPENDIX C: LOW PL INTENSITY STATE}

The low PL intensity state was observed in NCs with and without $\mathrm{CuSCN}$ in the film. In Fig. 7, we plot the number of NCs as a function of the total time in the low PL intensity state during the time trace of $100 \mathrm{~s}$ for NCs without (upper panel) and with CuSCN (lower panel). The low PL intensity state is significantly suppressed by CuSCN treatment. The low PL intensity state originates from positive trions.

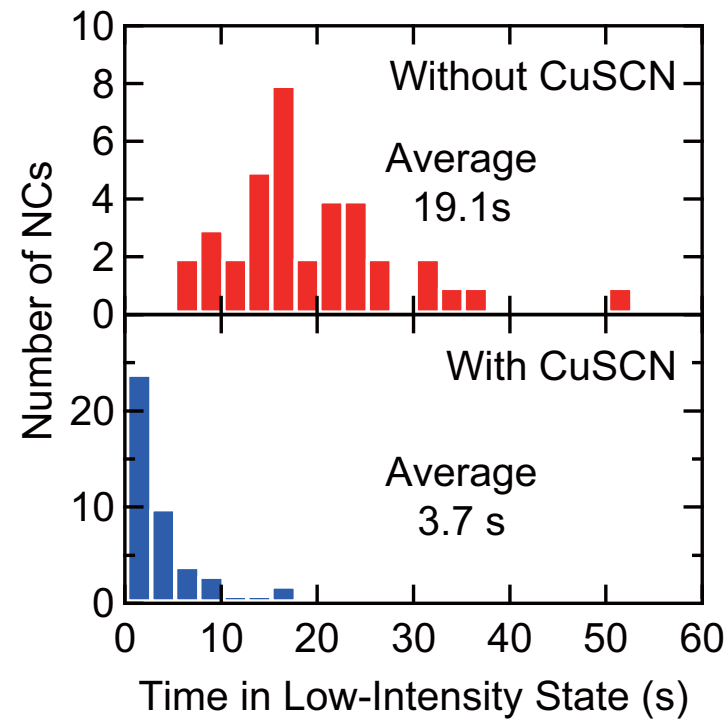

FIG. 7. Histograms of the total time in the low PL intensity state during the time trace of $100 \mathrm{~s}$ for single NCs without (upper panel) and with CuSCN (lower panel).
[1] M. M. Lee, J. Teuscher, T. Miyasaka, T. N. Murakami, and H. J. Snaith, Efficient hybrid solar cells based on mesosuperstructured organometal halide perovskites, Science 338, 643 (2012).

[2] H.-S. Kim, C.-R. Lee, J.-H. Im, K.-B. Lee, T. Moehl, A. Marchioro, S.-J. Moon, R. Humphry-Baker, J.-H. Yum, J. E. Moser, M. Grätzel, and N.-G. Park, Lead iodide perovskite sensitized all-solid-state submicron thin film mesoscopic solar cell with efficiency exceeding 9\%, Sci. Rep. 2, 591 (2012).

[3] Z.-K. Tan, R. S. Moghaddam, M. L. Lai, P. Docampo, R. Higler, F. Deschler, M. Price, A. Sadhanala, L. M. Pazos, D. Credgington, F. Hanusch, T. Bein, H. J. Snaith, and R. H. Friend, Bright light-emitting diodes based on organometal halide perovskite, Nat. Nanotechnol. 9, 687 (2014).

[4] G. Xing, N. Mathews, S. S. Lim, N. Yantara, X. Liu, D. Sabba, M. Grätzel, S. Mhaisalkar, and T. C. Sum, Low-temperature solution-processed wavelength-tunable perovskites for lasing, Nat. Mater. 13, 476 (2014).

[5] F. Deschler, M. Price, S. Pathak, L. E. Klintberg, D.-D. Jarausch, R. Higler, S. Hüttner, T. Leijtens, S. D. Stranks, H. J. Snaith, M. Atatüre, R. T. Phillips, and R. H. Friend, High photoluminescence efficiency and optically pumped lasing in solution-processed mixed halide perovskite semiconductors, J. Phys. Chem. Lett. 5, 1421 (2014).

[6] H. Tahara, T. Aharen, A. Wakamiya, and Y. Kanemitsu, Photorefractive effect in organic-inorganic hybrid perovskites and its application to optical phase shifter, Adv. Opt. Mater. 6, 1701366 (2018).

[7] Y. Yamada, T. Nakamura, M. Endo, A. Wakamiya, and Y. Kanemitsu, Photocarrier recombination dynamics in perovskite $\mathrm{CH}_{3} \mathrm{NH}_{3} \mathrm{PbI}_{3}$ for solar cell applications, J. Am. Chem. Soc. 136, 11610 (2014).
[8] E. M. Hutter, M. C. Gélvez-Rueda, A. Osherov, V. Bulović, F. C. Grozema, S. D. Stranks, and T. J. Savenije, Direct-indirect character of the bandgap in methylammonium lead iodide perovskite, Nat. Mater. 16, 115 (2017).

[9] S. D. Stranks, G. E. Eperon, G. Grancini, C. Menelaou, M. J. P. Alcocer, T. Leijtens, L. M. Herz, A. Petrozza, and H. J. Snaith, Electron-hole diffusion lengths exceeding 1 micrometer in an organometal trihalide perovskite absorber, Science $\mathbf{3 4 2}$, 341 (2013).

[10] G. Xing, N. Mathews, S. Sun, S. S. Lim, Y. M. Lam, M. Graätzel, S. Mhaisalkar, and T. C. Sum, Long-range balanced electron- and hole-transport lengths in organic-inorganic $\mathrm{CH}_{3} \mathrm{NH}_{3} \mathrm{PbI}_{3}$, Science 342, 344 (2013).

[11] T. Yamada, Y. Yamada, H. Nishimura, Y. Nakaike, A. Wakamiya, Y. Murata, and Y. Kanemitsu, Fast free-carrier diffusion in $\mathrm{CH}_{3} \mathrm{NH}_{3} \mathrm{PbBr}_{3}$ single crystals revealed by time-resolved one- and two-photon excitation photoluminescence spectroscopy, Adv. Electron. Mater. 2, 1500290 (2016).

[12] K. Miyata, T. L. Atallah, and X.-Y. Zhu, Lead halide perovskites: Crystal-liquid duality, phonon glass electron crystals, and large polaron formation, Sci. Adv. 3, e1701469 (2017).

[13] Y. Yamada, T. Yamada, L. Q. Phuong, N. Maruyama, H. Nishimura, A. Wakamiya, Y. Murata, and Y. Kanemitsu, Dynamic optical properties of $\mathrm{CH}_{3} \mathrm{NH}_{3} \mathrm{PbI}_{3}$ single crystals as revealed by one- and two-photon excited photoluminescence measurements, J. Am. Chem. Soc. 137, 10456 (2015).

[14] A. Kojima, K. Teshima, Y. Shirai, and T. Miyasaka, Organometal halide perovskites as visible-light sensitizers for photovoltaic cells, J. Am. Chem. Soc. 131, 6050 (2009).

[15] National Renewable Energy Laboratory Best Research-Cell Efficiencies, https://www.nrel.gov/pv/assets/pdfs/pv-efficiencies07-17-2018.pdf. 
[16] L. Zhang, X. Yang, Q. Jiang, P. Wang, Z. Yin, X. Zhang, H. Tan, Y. M. Yang, M. Wei, B. R. Sutherland, E. H. Sargent, and J. You, Ultra-bright and highly efficient inorganic based perovskite light-emitting diodes, Nat. Commun. 8, 15640 (2017).

[17] T. Umebayashi, K. Asai, T. Kondo, and A. Nakao, Electronic structures of lead iodide based low-dimensional crystals, Phys. Rev. B 67, 155405 (2003).

[18] W.-J. Yin, T. Shi, and Y. Yan, Unusual defect physics in $\mathrm{CH}_{3} \mathrm{NH}_{3} \mathrm{PbI}_{3}$ perovskite solar cell absorber, Appl. Phys. Lett. 104, 063903 (2014).

[19] R. E. Brandt, J. R. Poindexter, P. Gorai, R. C. Kurchin, R. L. Z. Hoye, L. Nienhaus, M. W. B. Wilson, J. A. Polizzotti, R. Sereika, R. Žaltauskas, L. C. Lee, J. L. MacManus-Driscoll, M. Bawendi, V. Stevanović, and T. Buonassisi, Searching for "defect-tolerant" photovoltaic materials: Combined theoretical and experimental screening, Chem. Mater. 29, 4667 (2017).

[20] L. C. Schmidt, A. Pertegás, S. González-Carrero, O. Malinkiewicz, S. Agouram, G. Mínguez Espallargas, H. J. Bolink, R. E. Galian, and J. Pérez-Prieto, Nontemplate synthesis of $\mathrm{CH}_{3} \mathrm{NH}_{3} \mathrm{PbBr}_{3}$ perovskite nanoparticles, J. Am. Chem. Soc. 136, 850 (2014).

[21] L. Protesescu, S. Yakunin, M. I. Bodnarchuk, F. Krieg, R. Caputo, C. H. Hendon, R. X. Yang, A. Walsh, and M. V. Kovalenko, Nanocrystals of cesium lead halide perovskites $\left(\mathrm{CsPb} X_{3}, X=\mathrm{Cl}, \mathrm{Br}\right.$, and $\left.\mathrm{I}\right)$ : Novel optoelectronic materials showing bright emission with wide color gamut, Nano Lett. 15, 3692 (2015).

[22] G. Nedelcu, L. Protesescu, S. Yakunin, M. I. Bodnarchuk, M. J. Grotevent, and M. V. Kovalenko, Fast anion-exchange in highly luminescent nanocrystals of cesium lead halide perovskites $\left(\mathrm{CsPb}_{3}, X=\mathrm{Cl}, \mathrm{Br}, \mathrm{I}\right)$, Nano Lett. 15, 5635 (2015).

[23] M. A. Hines and P. Guyot-Sionnest, Synthesis and characterization of strongly luminescing ZnS-capped CdSe nanocrystals, J. Phys. Chem. 100, 468 (1996).

[24] B. O. Dabbousi, J. Rodriguez-Viejo, F. V. Mikulec, J. R. Heine, H. Mattoussi, R. Ober, K. F. Jensen, and M. G. Bawendi, (CdSe)ZnS core-shell quantum dots: Synthesis and characterization of a size series of highly luminescent nanocrystallites, J. Phys. Chem. B 101, 9463 (1997).

[25] Q. A. Akkerman, G. Rainò, M. V. Kovalenko, and L. Manna, Genesis, challenges and opportunities for colloidal lead halide perovskite nanocrystals, Nature Mater. 17, 394 (2018).

[26] N. Yarita, H. Tahara, T. Ihara, T. Kawawaki, R. Sato, M. Saruyama, T. Teranishi, and Y. Kanemitsu, Dynamics of charged excitons and biexcitons in $\mathrm{CsPbBr}_{3}$ perovskite nanocrystals revealed by femtosecond transient-absorption and single-dot luminescence spectroscopy, J. Phys. Chem. Lett. 8, 1413 (2017).

[27] M. A. Becker, R. Vaxenburg, G. Nedelcu, P. C. Sercel, A. Shabaev, M. J. Mehl, J. G. Michopoulos, S. G. Lambrakos, N. Bernstein, J. L. Lyons, T. Stöferle, R. F. Mahrt, M. V. Kovalenko, D. J. Norris, G. Rainò, and Al. L. Efros, Bright triplet excitons in caesium lead halide perovskites, Nature (London) 553, 189 (2018).

[28] S. Yakunin, L. Protesescu, F. Krieg, M. I. Bodnarchuk, G. Nedelcu, M. Humer, G. De Luca, M. Fiebig, W. Heiss, and M. V. Kovalenko, Low-threshold amplified spontaneous emission and lasing from colloidal nanocrystals of caesium lead halide perovskites, Nat. Commun. 6, 8056 (2015).
[29] Z. Xiao, R. A. Kerner, L. Zhao, N. L. Tran, K. M. Lee, T.-W. Koh, G. D. Scholes, and B. P. Rand, Efficient perovskite light-emitting diodes featuring nanometre-sized crystallites, Nat. Photonics. 11, 108 (2017).

[30] H.-C. Wang, W. Wang, A.-C. Tang, H.-Y. Tsai, Z. Bao, T. Ihara, N. Yarita, H. Tahara, Y. Kanemitsu, S. Chen, and R.$\mathrm{S}$. Liu, High-performance $\mathrm{CsPb}_{1-x} \mathrm{Sn}_{x} \mathrm{Br}_{3}$ perovskite quantum dots for light-emitting diodes, Angew. Chem. Int. Ed. 56, 13650 (2014).

[31] V. I. Klimov, Multicarrier interactions in semiconductor nanocrystals in relation to the phenomena of Auger recombination and carrier multiplication, Annu. Rev. Condens. Matter Phys. 5, 285 (2014).

[32] Y. Kanemitsu, Multiple exciton generation and recombination in carbon nanotubes and nanocrystals, Acc. Chem. Res. 46, 1358 (2013).

[33] J. M. Pietryga, Y.-S. Park, J. Lim, A. F. Fidler, W. K. Bae, S. Brovelli, and V. I. Klimov, Spectroscopic and device aspects of nanocrystal quantum dots, Chem. Rev. 116, 10513 (2016).

[34] N. S. Makarov, S. Guo, O. Isaienko, W. Liu, I. Robel, and V. I. Klimov, Spectral and dynamical properties of single excitons, biexcitons, and trions in cesium-lead-halide perovskite quantum dots, Nano Lett. 16, 2349 (2016).

[35] J. A. Castañeda, G. Nagamine, E. Yassitepe, L. G. Bonato, O. Voznyy, S. Hoogland, A. F. Nogueira, E. H. Sargent, C. H. B. Cruz, and L. A. Padilha, Efficient biexciton interaction in perovskite quantum dots under weak and strong confinement, ACS Nano 10, 8603 (2016).

[36] S. Nakahara, H. Tahara, G. Yumoto, T. Kawawaki, M. Saruyama, R. Sato, T. Teranishi, and Y. Kanemitsu, Suppression of trion formation in $\mathrm{CsPbr}_{3}$ perovskite nanocrystals by postsynthetic surface modification, J. Phys. Chem. C 122, 22188 (2018).

[37] M. Nirmal, B. O. Dabbousi, M. G. Bawendi, J. J. Macklin, J. K. Trautman, T. D. Harris, and L. E. Brus, Fluorescence intermittency in single cadmium selenide nanocrystals, Nature (London) 383, 802 (1996).

[38] Al. L. Efros and D. J. Nesbitt, Origin and control of blinking in quantum dots, Nat. Nanotechnol. 11, 661 (2016).

[39] K. Wu, Y.-S. Park, J. Lim, and V. I. Klimov, Towards zerothreshold optical gain using charged semiconductor quantum dots, Nature Nanotechnol. 12, 1140 (2017).

[40] G. Yumoto, H. Tahara, T. Kawawaki, M. Saruyama, R. Sato, T. Teranishi, and Y. Kanemitsu, Hot biexciton effect on optical gain in $\mathrm{CsPbI}_{3}$ perovskite nanocrystals, J. Phys. Chem. Lett. 9, 2222 (2018).

[41] Y. Wang, M. Zhi, Y.-Q. Chang, J.-P. Zhang, and Y. Chan, Stable, ultralow threshold amplified spontaneous emission from $\mathrm{CsPbBr}_{3}$ nanoparticles exhibiting trion gain, Nano lett. 18, 4976 (2018).

[42] L. Protesescu, S. Yakunin, M. I. Bodnarchuk, F. Bertolotti, N. Masciocchi, A. Guagliardi, and M. V. Kovalenko, Monodisperse formamidinium lead bromide nanocrystals with bright and stable green photoluminescence, J. Am. Chem. Soc. 138, 14202 (2016)

[43] N. Yarita, H. Tahara, M. Saruyama, T. Kawawaki, R. Sato, T. Teranishi, and Y. Kanemitsu, Impact of Postsynthetic Surface modification on photoluminescence intermittency in formamidinium lead bromide perovskite nanocrystals, J. Phys. Chem. Lett. 8, 6041 (2017). 
[44] N. Hiroshige, T. Ihara, and Y. Kanemitsu, Simultaneously measured photoluminescence lifetime and quantum yield of two-photon cascade emission on single CdSe/ZnS nanocrystals, Phys. Rev. B 95, 245307 (2017).

[45] N. Hiroshige, T. Ihara, M. Saruyama, T. Teranishi, and Y. Kanemitsu, Coulomb-enhanced radiative recombination of biexcitons in single giant-shell CdSe/CdS core/shell nanocrystals, J. Phys. Chem. Lett. 8, 1961 (2017).

[46] G. Nair, J. Zhao, and M. G. Bawendi, Biexciton quantum yield of single semiconductor nanocrystals from photon statistics, Nano Lett. 11, 1136 (2011).

[47] S. Ye, W. Sun, Y. Li, W. Yan, H. Peng, Z. Bian, Z. Liu, and C. Huang, CuSCN-based inverted planar perovskite solar cell with an average PCE of 15.6\%, Nano Lett. 15, 3723 (2015).

[48] J. Liu, S. K. Pathak, N. Sakai, R. Sheng, S. Bai, Z. Wang, and H. J. Snaith, Identification and mitigation of a critical interfacial instability in perovskite solar cells employing copper thiocyanate hole-transporter, Adv. Mater. Interfaces 3, 1600571 (2016).

[49] J. D. Rinehart, A. M. Schimpf, A. L. Weaver, A. W. Cohn, and D. R. Gamelin, Photochemical electronic doping of colloidal CdSe nanocrystals, J. Am. Chem. Soc. 135, 18782 (2013).
[50] K. Wu, J. Lim, and V. I. Klimov, Superposition principle in Auger recombination of charged and neutral multicarrier states in semiconductor quantum dots, ACS Nano 11, 8437 (2017).

[51] P. Spinicelli, S. Buil, X. Quélin, B. Mahler, B. Dubertret, and J.P. Hermier, Bright and Grey States in CdSe-CdS Nanocrystals Exhibiting Strongly Reduced Blinking, Phys. Rev. Lett. 102, 136801 (2009).

[52] P. P. Jha and P. Guyot-Sionnest, Trion decay in colloidal quantum dots, ACS Nano 3, 1011 (2009).

[53] D. E. Gómez, J. Van Embden, P. Mulvaney, M. J. Fernée, and H. Rubinsztein-Dunlop, Exciton-trion transitions in single CdSe-CdS core-shell nanocrystals, ACS Nano 3, 2281 (2009).

[54] Y.-S. Park, W. K. Bae, J. M. Pietryga, and V. I. Klimov, Auger recombination of biexcitons and negative and positive trions in individual quantum dots, ACS Nano 8, 7288 (2014).

[55] T. Handa, T. Yamada, H. Kubota, S. Ise, Y. Miyamoto, and Y. Kanemitsu, Photocarrier recombination and injection dynamics in long-term stable lead-free $\mathrm{CH}_{3} \mathrm{NH}_{3} \mathrm{SnI}_{3}$ perovskite thin films and solar cells, J. Phys. Chem. C 121, 16158 (2017). 